MS02-04

\section{Ctrl-D, a versatile tool for analyzing processed diffraction data}

Fabio Dall'Antonia ${ }^{1}$, Gleb Bourenkov ${ }^{1}$, Thomas Schneider ${ }^{1}$

1. European Molecular Biology Laboratory, Hamburg Unit, Hamburg, Germany

email: fabio.dallantonia@embl-hamburg.de

Good native data are an asset for phasing, because they allow you to obtain and improve solutions to the phase problem even in the absence of a suitable homologous template. The question often explored in Molecular Replacement (MR) is: Is the model good enough to solve the structure? Here we explore the reverse: Are the data good enough for the distant homolog model to succeed? ARCIMBOLDO_SHREDDER (1) derives compact model fragments starting from a distant homolog template, evaluating their performance in a process driven by the experimental data, provided that a resolution of at least $2.5 \AA$ is available. This method uses partial fragments that need to be very accurate. The following stages can be distinguished:

- Partition and annotation of the template: The template model is dissected into fragments that will be disassembled to give the model additional degrees of freedom.

- Generation of the models: The size of the fragments is derived from a target eLLG, set to be large enough to find correct solutions, but small enough to generate potentially accurate fragments.

- Evaluation against the likelihood rotation-function target

- Gyre refinement (2): The orientation and relative translation of the rigid groups identified in the first step are refined against the rotation-function target.

- Translation search

- Packing test

- Refinement: Depending on the data and the expected deviation of the models, a set of different strategies can be trialed at this stage: refinement of the r.m.s. error attributed to the model, superposition of the original template on each placed fragment with trimming and refinement, gimble refinement against the likelihood translation-function target subdividing the model into the same rigid groups as in gyre, or phaser's likelihood-based pruning.

- Phase combination: Consistent phase sets can be combined with ALIXE in order to complete partial solutions and increase their information content.

- Density modification and autotracing for expansion of the substructure to a full solution: The single or combined phase sets are used to calculate starting maps for iterative density modification and autotracing with SHELXE.

The method described has been successful in solving previously unknown structures, including Slt (3), a lytic transglycosylase from the pathogen Pseudomonas aeruginosa. Moreover, it has helped to increase our knowledge about how to best exploit the available data and models in the context of fragment-based MR. In this presentation, both the method and its application to different cases will be discussed.

References:

[1.] R. W. Grosse-Kunstleve, et al. (2002). J. Appl. Cryst. 35, 126-136.

[2.] S. L. S. Storm et al. (2017). J. Synchrotron Rad. 24, 19-28.

[3.] T. Pape \& T. R. Schneider (2004). J. Appl. Cryst. 37, 843-844.

Keywords: Data-analysis, SSX, SAD-Phasing 